\title{
Diversidade florística do bairro Nossa Senhora das Dores em Santa Maria, $\mathbf{R S}^{(1)}$
}

\author{
NATALIA TEIXEIRA SCHWAB (2), LEONITA BEATRIZ GIRARDI (2), \\ MAURICIO NEUHAUS (2), FERNANDA ALICE ANTONELLO LONDERO BACKES (2), \\ ROGÉRIO ANTÔNIO BELLÉ (2), JANINE FARIAS MENEGAES (2)
}

\begin{abstract}
RESUMO
As áreas arborizadas existentes no contexto urbano contribuem significativamente para a melhoria da qualidade de vida da sociedade. Diagnosticar essas áreas é importante para a elaboração do planejamento da arborização de uma cidade. Assim, o objetivo do trabalho foi identificar, por meio de um inventário, e diagnosticar a qualidade das espécies vegetais no bairro Nossa Senhora das Dores em Santa Maria, RS, realizado no período de fevereiro a abril de 2010. Foram encontradas 99 espécies botânicas distribuídas em 47 famílias, sendo o bairro considerado com ampla diversidade vegetal. As espécies Lagerstroemia indica L., Tipuana tipu (Benth.)Kuntze e Syagrus romanzoffiana (Cham.) Glassman apresentaram maior frequência nas vias. Verificou-se a presença de várias espécies inadequadas à arborização das calçadas, como Ficus benjamina L., Inga marginata Willd., Hovenia dulcis Thunb., Melia azedarach L., entre outras. Sugere-se uma adequação e renovação de algumas espécies para o bairro Nossa Senhora das Dores a fim de proporcionar aos moradores um melhor aproveitamento do verde urbano.
\end{abstract}

Palavras-chave: Arborização urbana; espécies vegetais; qualidade de vida.

\begin{abstract}
Floristic diversity of Nossa Senhora das Dores neighborhood in Santa Maria, RS

Wooded areas in the urban context contribute significantly for improving quality of society's life. Diagnosing such areas is very important for the development of future afforestation planning of a city. Thus, the objective of this work was to identify, by means of a quali-quantitative inventory, the plant species in Nossa Senhora das Dores neighborhood, in Santa Maria, RS, in the period from February to April, 2010. We found 99 botanical species distributed in 47 families, so the neighborhood can be considered as having great plant diversity. The species Lagerstroemia indica L., Tipuana tipu (Benth.) Kuntze and Syagrus romanzoffiana (Cham.) Glassman showed higher frequency on the public roads of the neighborhood. Many species were inadequate to the afforestation of sidewalks, such as, Ficus benjamina L., Inga marginata Willd., Hovenia dulcis Thunb., Melia azedarach L., among others. Suitability and renewal of some species are recommended to the Nossa Senhora das Dores neighborhood so that the dwellers can better enjoy the urban green.

Keywords: Urban forest; plant species; life quality.
\end{abstract}

\section{INTRODUÇÃO}

O planejamento da arborização no meio urbano tem como fator decisivo a escolha adequada da espécie, para minimizar problemas futuros, como a poda e danos provocados pelas raízes. Durante a fase de planejamento, vários critérios e fatores devem ser considerados, como a largura de calçadas, a presença de fiação aérea, as tubulações subterrâneas de esgoto e a drenagem (GONÇALVES e PAIVA, 2004).

Em muitas situações, o planejamento urbano deixa de incluir a arborização, permitindo que iniciativas particulares pontuais ocupem o espaço. Entretanto, quando espécies sem compatibilidade com o local são plantadas, perde-se a eficácia da arborização em transmitir conforto físico e psíquico, acarretando infortúnios e transtornos (SILVA FILHO et al., 2002). Grande parte dos problemas enfrentados na arborização urbana está ligada ao desconhecimento das espécies estabelecidas, evidenciando, dessa maneira, que a adequada seleção contribui para o sucesso do empreendimento. Assim, o objetivo do trabalho foi identificar, por meio de um inventário, as espécies vegetais presentes no bairro Nossa Senhora das Dores em Santa Maria, RS.

\section{MATERIAL E MÉTODOS}

O inventário florístico foi realizado no município de Santa Maria, RS (latitude $29^{\circ} 43^{\prime} \mathrm{S}$, longitude $53^{\circ} 43^{\prime} \mathrm{W}$ e altitude $95 \mathrm{~m}$ ), no bairro Nossa Senhora das Dores, que se localiza na região nordeste da cidade e possui uma área de $1,0873 \mathrm{~km}^{2}$, que corresponde a $0,89 \%$ da extensão total $\left(121,84 \mathrm{~km}^{2}\right)$. O estudo foi realizado no período de fevereiro a abril de 2010 . A escolha do bairro justifica-se por possuir uma arborização considerável e intensa urbanização.

Todas as espécies vegetais foram identificadas pelo nome comum, nome científico, origem e família botânica, por meio de levantamento tipo censo, nas 31 vias públicas do bairro. As espécies vegetais foram classificadas quanto:

a) à estimativa da altura total quando adulta (pequena $\leq 1,0 \mathrm{~m}$, média -4 a $6 \mathrm{~m}$ e grande $->6 \mathrm{~m}$ );

b) ao estado geral da planta (ótimo - árvore vigorosa e sadia, sem sinais aparentes de ataque de insetos, doenças ou injúrias mecânicas, pequena ou nenhuma necessidade de manutenção, forma e arquitetura características da espécie; bom - médias condições de vigor e saúde, necessita de pequenos reparos ou poda, apresenta leve descaracterização da forma e sinais visíveis

\footnotetext{
(1) Trabalho recebido para publicação em 23/01/2013 e aprovado em 21/08/2014

(2) Departamento de Fitotecnia, Universidade Federal de Santa Maria. Av. Roraima n ${ }^{\text {}} 1000$ - Cidade Universitária - Bairro Camobi - Santa Maria - RS -CEP: 97105-900. (fernanda@backes.com)
} 
de problemas fisiológicos; regular - apresenta estado geral em início de senescência, apresenta danos mecânicos, danos no tronco, desequilíbrio na arquitetura e problemas fisiológicos; e péssimo - avançado estado de senescência, injúrias graves no tronco e poda descaracterizando a arquitetura do vegetal, plantas sem perspectivas de recuperação) (adaptado de SILVA FILHO et al., 2002);

c) ao aspecto da copa (normal - estrutura normal da espécie segundo a literatura; medianamente deformada - copa com deformações que alteram a sua estrutura normal, causadas por podas mal conduzidas; e deformada - quando a estrutura é bastante alterada, assimétrica, em decorrência de podas drásticas ou mal conduzidas);

d) à ocorrência de danos na calçada e em conflito com a fiação.

A frequência relativa de cada espécie foi calculada pela razão entre o número de indivíduos da espécie e o número total de indivíduos dentro de cada grupo (arbóreas, palmeiras, arbustos e coníferas), multiplicada por 100. O índice de riqueza de espécies foi calculado segundo Simpson (1949) apud SILVA FILHO et al., (2002), com a equação: $d=(S-1) / \log _{0} N$, em que: $\mathrm{d}=$ riqueza de espécies; $\mathrm{S}=$ número de espécies; $\mathrm{N}=$ número total de indivíduos.

\section{RESULTADOS E DISCUSSÃO}

No levantamento florístico foram encontrados 1.059 indivíduos, sendo 101 espécies distribuídas em 50 famílias botânicas (Tabela 1). Verificou-se que há predomínio de espécies exóticas, correspondente a 69,3\% do total, enquanto a ocorrência de espécies nativas correspondeu a apenas 30,7\%. Observa-se grande desequilíbrio entre espécies exóticas e nativas, provavelmente devido à preferência e ao gosto popular, pois a grande maioria dos indivíduos implantados no local foi introduzida pela população. Considerando esses valores para a área de 1,08 $\mathrm{km}^{2}$, pode-se considerar o espaço arborizado das ruas e da avenida com uma grande diversidade botânica de famílias e de espécies. O índice de riqueza de Simpson mostrou um valor de 33,06.

As famílias de maior ocorrência em número de exemplares foram Lytraceae, Fabaceae e Arecaceae, respectivamente com $21,16 \%, 17,65 \%$ e $17,18 \%$. A primeira família está representada, exclusivamente, por extremosas, enquanto a segunda, por 16 diferentes espécies e a terceira, por oito espécies de palmeiras.

Quanto à frequência das espécies, observou-se grande variabilidade, e as nove mais frequentes estão representadas na Figura 1, representando 63,16\% da vegetação viária do bairro. As espécies mais frequentes foram Lagerstroemia indica $(21,47 \%)$, seguida de Syagrus romanzoffiana $(13,97 \%)$ e de Tipuana tipu (10,76\%). As espécies com menor frequência encontradas no levantamento são aquelas com ocorrência de apenas um único exemplar (0,09\%), e corresponderam a $37,6 \%$ da variabilidade de espécies presentes. Já 12,8\% das espécies possuem dois exemplares e 9,9\%, três exemplares.

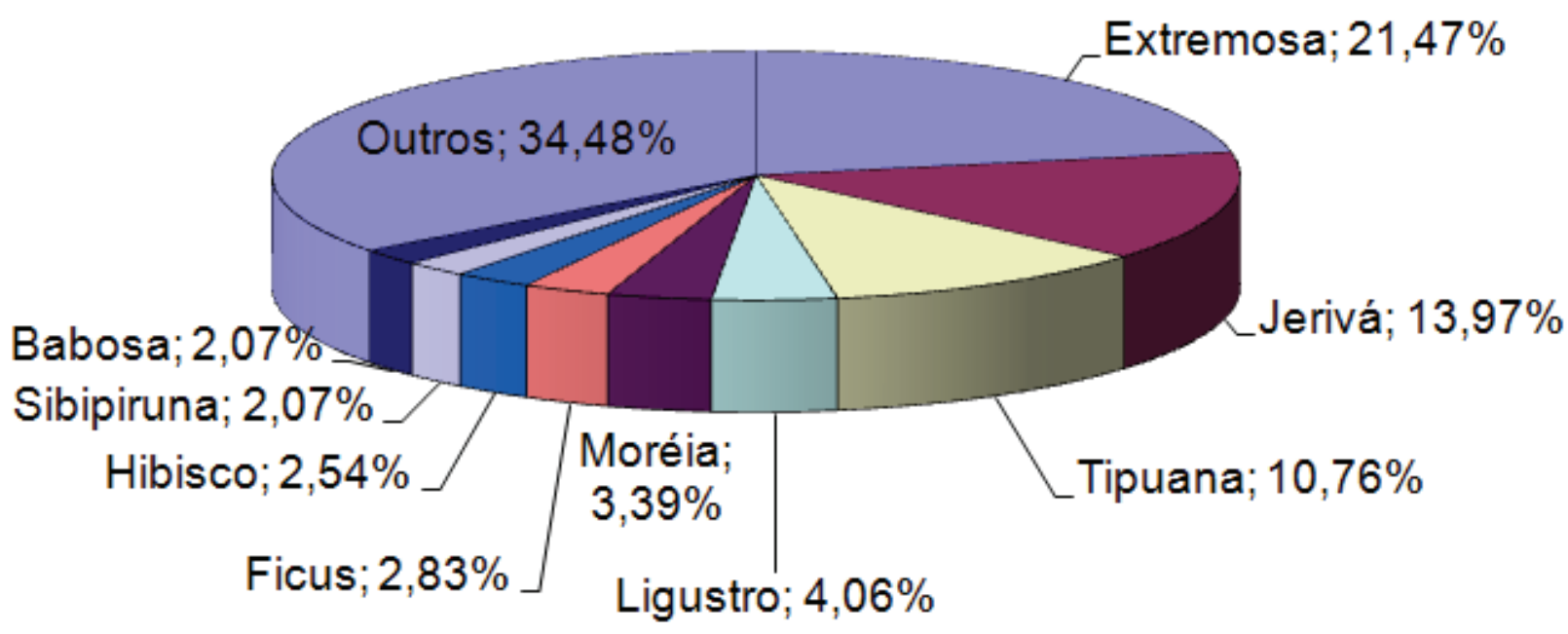

Figura 1. Distribuição percentual das espécies de maior ocorrência nas vias públicas do bairro Nossa Senhora das Dores. Santa Maria, 2010.

Figure 1. Percentual distribution of the most common species on public roads in Nossa Senhora das Dores neighborhood. Santa Maria, RS, 2010.

A extremosa foi a espécie dominante do bairro, onde cinco vias concentram $94,7 \%$ dos indivíduos. Nelas, há uma variação entre $51,2 \%$ e $93,4 \%$ dessa espécie, o que denota que, num determinado momento do passado, essas vias sofreram arborização maciça, cujo sucesso variou conforme o local. Os exemplares, à medida que foram eliminados, pouco a pouco foram sendo substituídos segundo o gosto da vizinhança. A arborização com uma única espécie tornou o ambiente pesado, especialmente no momento floração, pois as espécies implantadas no local apresentam praticamente uma única cor na florada, resultando na monocromia do pink. A diversidade de espécies na arborização de vias não implica plantio aleatório; no entanto, recomenda-se manter uniformidade dentro das quadras ou mesmo das ruas e avenidas, utilizando-se uma ou duas espécies (PIVETTA e SILVA FILHO, 2002). 
Quanto a Syagrus romanzoffiana, esta espécie está concentrada praticamente em uma única avenida, fazendo parte do canteiro central e rótulas, onde, em alguns locais, sua densidade é excessiva. Ribeiro et al. (2007), avaliando a arborização de praças de Vinhedos/SP, verificaram maior ocorrência desta espécie com 31,94\%.

A frequência de ocorrência das espécies que compõem a arborização no Bairro Nossa Senhora das Dores apresentase desequilibrada, não resultando em uma composição harmoniosa da vegetação. A harmonia da vegetação é um parâmetro que deve ser considerado durante o planejamento da arborização de vias públicas, devendo apresentar valores entre 10 e $15 \%$, e a predominância de um grupo de espécies pode ocasionar riscos quanto à sua longevidade e ao ataque de pragas e doenças (GREY e DENEKE, 1978 apud MILANO e DALCIN, 2000).

Deste modo, dez espécies podem ser suficientes para compor o conjunto da vegetação de um bairro, o que não se observou neste caso. A má frequência é representada por espécies inadequadas para este tipo de arborização, como tipuana, fícus-benjamina e ingá (Tabela 1). Além disso, a presença de um grande número de espécies com apenas um único exemplar causa desorganização na distribuição dos indivíduos, levando a uma situação de arborização caótica.

Tabela 1. Identificação, origem nativa $(\mathrm{N})$ ou exótica (E), família, número de exemplares e frequência relativa (FR) da vegetação viária do Bairro Nossa Senhora das Dores em Santa Maria, RS, 2010

Table 1. Identification, native $(N)$ or exotic (E), family, number and relative frequency (RF) of the road vegetation occurring in Nossa Senhora das Dores neighborhood in Santa Maria, RS, 2010

\begin{tabular}{|c|c|c|c|c|c|}
\hline Nome vulgar & Nome científico & Origem & Família & Número & FR \\
\hline Extremosa & Lagerstroemia indica $\mathrm{L}$. & E & Lythraceae & 227 & 21,47 \\
\hline Jerivá & Syagrus romanzoffiana (Cham.) Glassm. & $\mathrm{N}$ & Arecaceae & 148 & 13,97 \\
\hline Tipuana & Tipuana tipu (Benth.) Kuntze & $\mathrm{E}$ & Fabaceae & 114 & 10,76 \\
\hline Ligustro & Ligustrum lucidum W.T. Aiton & E & Oleaceae & 43 & 4,06 \\
\hline Moréia & Dietes bicolor Sweet ex Klatt & E & Iridaceae & 36 & 3,39 \\
\hline Ficus-benjamina & Ficus benjamina L. & E & Moraceae & 30 & 2,83 \\
\hline Hibisco & Hibiscus rosa-sinensis L. & $\mathrm{E}$ & Malvaceae & 27 & 2,54 \\
\hline Sibipiruna & Caesalpinia peltophoroides Benth. & $\mathrm{N}$ & Fabaceae & 25 & 2,36 \\
\hline Babosa & Aloe arborescens Mill. & $\mathrm{E}$ & Asphodelaceae & 22 & 2,07 \\
\hline Seafórtia & $\begin{array}{l}\text { Archontophoenix alexandrae H. wendl. \& } \\
\text { Drude }\end{array}$ & $\mathrm{E}$ & Arecaceae & 21 & 1,98 \\
\hline Escova-de-garrafa & $\begin{array}{l}\text { Callistemon viminalis (Sol. Ex Gaertn.) G. } \\
\text { Don ex Loud. }\end{array}$ & $\mathrm{E}$ & Myrtaceae & 20 & 1,88 \\
\hline Agave & Agave angustifolia Haw. & $\mathrm{E}$ & Agavaceae & 18 & 1,69 \\
\hline Ingá-feijão & Inga marginata Willd. & $\mathrm{N}$ & Fabaceae & 18 & 1,69 \\
\hline Ipê-amarelo & $\begin{array}{c}\text { Handroanthus chrysotrichus (Mart. Ex A. } \\
\text { DC.) Mattos }\end{array}$ & $\mathrm{N}$ & Bignoniaceae & 17 & 1,60 \\
\hline Uva-do-japão & Hovenia dulcis Thunb. & $\mathrm{E}$ & Rhamnaceae & 16 & 1,51 \\
\hline Agave-dragão & Agave attenuata Salm-Dyck & $\mathrm{E}$ & Agavaceae & 15 & 1,41 \\
\hline Cinamomo & Melia azedarach L. & E & Meliaceae & 15 & 1,41 \\
\hline Pingo-d'ouro & Duranta erecta L. "Gold Mound" & $\mathrm{N}$ & Verbenaceae & 13 & 1,22 \\
\hline Canela-de-rama & Cinnaтотит zeylanicum Nees & $\mathrm{E}$ & Lauraceae & 12 & 1,13 \\
\hline Ipê-roxo & Handroanthus heptaphyllus (Vell.) Mattos & $\mathrm{N}$ & Bignoniaceae & 12 & 1,13 \\
\hline Jacarandá & Jacaranda mimosifolia D. Don & $\mathrm{E}$ & Bignoniaceae & 10 & 0,94 \\
\hline Limoeiro & Citrus x limonia Osbeck & E & Rutaceae & 8 & 0,75 \\
\hline Flamboyant & Delonix regia (Bojer ex Hook.) Raf. & $\mathrm{E}$ & Fabaceae & 8 & 0,75 \\
\hline Goiabeira & Psidium guajava $\mathrm{L}$. & $\mathrm{N}$ & Myrtaceae & 8 & 0,75 \\
\hline Jambolão & Syzygium cumini (L.) Skeels & $\mathrm{E}$ & Myrtaceae & 8 & 0,75 \\
\hline Laranjeira & Citrus $x$ sinensis (L.) Osbeck & $\mathrm{E}$ & Rutaceae & 7 & 0,66 \\
\hline Cordiline & Cordilyne terminalis (L.) Kunth & $\mathrm{E}$ & Laxmanniaceae & 6 & 0,56 \\
\hline Canafístula & Peltophorum dubium (Spreng.) Taub. & $\mathrm{N}$ & Fabaceae & 6 & 0,56 \\
\hline Álamo & Populus nigra var. stricta & $\mathrm{E}$ & Salicaceae & 6 & 0,56 \\
\hline Ameixeira & Prunus domestica L. & $\mathrm{E}$ & Rosaceae & 6 & 0,56 \\
\hline Nandina & Nandina domestica Thunb. & $\mathrm{E}$ & Berberidaceae & 5 & 0,47 \\
\hline Roseira & Rosa x grandiflora Hort. & E & Rosaceae & 5 & 0,47 \\
\hline Aroeira-vermelha & Schinus terebinthifolius Raddi & $\mathrm{N}$ & Anacardiaceae & 5 & 0,47 \\
\hline Corticeira-do-banhado & Erythrina crista-galii L. & $\mathrm{N}$ & Fabaceae & 4 & 0,37 \\
\hline Guajuvira & Patagonula americana $\mathrm{L}$. & $\mathrm{N}$ & Boraginaceae & 4 & 0,37 \\
\hline Tamareira-anã & Phoenix roebelenii O'Brien & E & Arecaceae & 4 & 0,37 \\
\hline
\end{tabular}




\begin{tabular}{|c|c|c|c|c|c|}
\hline Araçá & Psidium cattleianum Sabine & E & Myrtaceae & 4 & 0,37 \\
\hline Piracanta & Pyracantha coccinea M. Roem. & $\mathrm{E}$ & Rosaceae & 4 & 0,37 \\
\hline Tuia-da-china & Thuja orientalis L. & $\mathrm{E}$ & Cupressaceae & 4 & 0,37 \\
\hline Pita & Yucca guatemalensis Baker & E & Agavaceae & 4 & 0,37 \\
\hline Chuva-de-ouro & Cassia fistula L. & $\mathrm{E}$ & Fabaceae & 3 & 0,28 \\
\hline Palmeira-triangular & Dypsis decaryi (Jum.) Beentje \& J. Dransf. & $\mathrm{E}$ & Arecaceae & 3 & 0,28 \\
\hline Pitanga & Eugenia uniflora L. & $\mathrm{N}$ & Myrtaceae & 3 & 0,28 \\
\hline Coroa-de-cristo & $\begin{array}{c}\text { Euphorbia milii var. hislopii (N. E. Br.) } \\
\text { Leandri }\end{array}$ & E & Euphorbiaceae & 3 & 0,28 \\
\hline Grevilea-robusta & Grevillea robusta A. Cunn. Ex. R. Br. & E & Proteaceae & 3 & 0,28 \\
\hline Açoita-cavalo & Luehea divaricata Mart. & $\mathrm{N}$ & Tiliaceae & 3 & 0,28 \\
\hline Amoreira & Morus nigra L. & E & Moraceae & 3 & 0,28 \\
\hline Tamareira-das-canárias & Phoenix canariensis Hort. Ex Chabaud & E & Arecaceae & 3 & 0,28 \\
\hline Scheflera & Schefflera arboricola (Hayata) Merr. & $\mathrm{E}$ & Araliaceae & 3 & 0,28 \\
\hline Agulha-de-adão & Yucca filamentosa $\mathrm{L}$. & E & Agavaceae & 3 & 0,28 \\
\hline Ariticum & Annona crassiflora Mart. & $\mathrm{N}$ & Annonaceae & 2 & 0,18 \\
\hline Araucária & Araucaria columnaris (Forst.) Hook. & $\mathrm{E}$ & Araucariaceae & 2 & 0,18 \\
\hline Manacá-de-cheiro & Brunfelsia uniflora (Pohl) D. Don & $\mathrm{N}$ & Solanaceae & 2 & 0,18 \\
\hline Pau-ferro & $\begin{array}{c}\text { Caesalpinia ferrea Mart. Ex Tul. var. } \\
\text { leiostachya Benth. }\end{array}$ & $\mathrm{N}$ & Fabaceae & 2 & 0,18 \\
\hline Falso-barbatimão & Cassia leptophylla Vog. & $\mathrm{N}$ & Fabaceae & 2 & 0,18 \\
\hline Timbaúva & $\begin{array}{c}\text { Enterolobium contortisiliquum (Vell.) } \\
\text { Morong }\end{array}$ & $\mathrm{N}$ & Fabaceae & 2 & 0,18 \\
\hline Hibisco-mutabilis & Hibiscus mutabilis L. & E & Malvaceae & 2 & 0,18 \\
\hline Mangueira & Mangifera indica $\mathrm{L}$. & E & Anacardiaceae & 2 & 0,18 \\
\hline Angico & Parapiptadenia rigida (Benth.) Brenan & $\mathrm{N}$ & Fabaceae & 2 & 0,18 \\
\hline Gerânio & Pelargonium x hortorum L. H. Bailey & $\mathrm{E}$ & Geraniaceae & 2 & 0,18 \\
\hline Azaléia & Rhododendron simsii Planch. & $\mathrm{E}$ & Ericaceae & 2 & 0,18 \\
\hline Coleus & Solenostemon scutellarioides (L.) Codd & E & Lamiaceae & 2 & 0,18 \\
\hline Tibouchina & Tibouchina granulosa Cogn. & $\mathrm{N}$ & Melastomaceae & 2 & 0,18 \\
\hline Pata-de-vaca & Bauhinia forficata Link & $\mathrm{N}$ & Fabaceae & 1 & 0,09 \\
\hline Três-marias & Bougainvillea spectabilis Willd. & $\mathrm{N}$ & Nyctaginaceae & 1 & 0,09 \\
\hline Butiá & $\begin{array}{c}\text { Butia odorata (Barb. Rodr.) Noblick \& } \\
\text { Lorenzi }\end{array}$ & $\mathrm{N}$ & Arecaceae & 1 & 0,09 \\
\hline Falso pau-brasil & Caesalpinea spinosa (Molina) Kuntze & $\mathrm{E}$ & Fabaceae & 1 & 0,09 \\
\hline Caliandra & Calliandra brevipes Benth. & $\mathrm{N}$ & Fabaceae & 1 & 0,09 \\
\hline Camélia & Camellia japonica $\mathrm{L}$. & $\mathrm{E}$ & Theaceae & 1 & 0,09 \\
\hline Mamoeiro & Carica papaya $\mathrm{L}$. & $\mathrm{E}$ & Caricaceae & 1 & 0,09 \\
\hline Noz-pecã & Carya illinoinensis (Wang.) K. Koch & $\mathrm{E}$ & Juglandaceae & 1 & 0,09 \\
\hline Carvalhinho & Casearia sylvestris $\mathrm{Sw}$. & $\mathrm{N}$ & Flacourtiaceae & 1 & 0,09 \\
\hline Paineira & Ceiba speciosa (A. St.-Hil.) Ravenna & $\mathrm{N}$ & Bombacaceae & 1 & 0,09 \\
\hline Celósia & Celosia cristata $\mathrm{L}$. & E & Amaranthaceae & 1 & 0,09 \\
\hline Cipreste-dourado & $\begin{array}{c}\text { Chamaecyparis obtusa (Siebold \& Zuccc.) } \\
\text { Endl. var. crippsii }\end{array}$ & $\mathrm{E}$ & Cupressaceae & 1 & 0,09 \\
\hline Lima & Citrus aurantifolia (Christm.) Swingle & E & Rutaceae & 1 & 0,09 \\
\hline Maranta-cinza & Ctenanthe setosa Eichler & $\mathrm{E}$ & Marantaceae & 1 & 0,09 \\
\hline Criptoméria & $\begin{array}{c}\text { Cryptomeria japonica (L. f.) D. Don var. } \\
\text { elegans }\end{array}$ & $\mathrm{E}$ & Taxodiaceae & 1 & 0,09 \\
\hline Cipreste-italiano & Cupressus sempervirens var. stricta & E & Cupressaceae & 1 & 0,09 \\
\hline Caquizeiro & Diospyros kaki L. f. & E & Ebenaceae & 1 & 0,09 \\
\hline Areca-bambu & Dypsis lutescens $\mathrm{H}$. Wendl. & $\mathrm{E}$ & Arecaceae & 1 & 0,09 \\
\hline Figueira-vermelha & Ficus auriculata Lour. & E & Moraceae & 1 & 0,09 \\
\hline Figo & Ficus carica L. & E & Moraceae & 1 & 0,09 \\
\hline Figueira-lacerdinha & Ficus microcarpa L. f. & E & Moraceae & 1 & 0,09 \\
\hline Furcrea & Furcraea foetida (L.) Haw. & E & Agavaceae & 1 & 0,09 \\
\hline Ipê-amarelo-da-serra & Handroanthus albus (Cham.) Mattos & $\mathrm{N}$ & Bignoniaceae & 1 & 0,09 \\
\hline Lantana & Lantana camara $\mathrm{L}$ & E & Verbenaceae & 1 & 0,09 \\
\hline
\end{tabular}




\begin{tabular}{|c|c|c|c|c|c|}
\hline Espirradeira & Nerium oleander L. & $\mathrm{E}$ & Apocynaceae & 1 & 0,09 \\
\hline Palma-miúda & Nopalea cochenillifera (L.) Salm-Dyck & $\mathrm{E}$ & Cactaceae & 1 & 0,09 \\
\hline Abacateiro & Persea americana Mill. & $\mathrm{E}$ & Lauraceae & 1 & 0,09 \\
\hline Umbu & Phytolacca dioica L. & & Phytolaccaceae & 1 & 0,09 \\
\hline Pinus & Pinus sp. & $\mathrm{E}$ & Pinaceae & 1 & 0,09 \\
\hline Pitospóro undulado & Pittosporum undulatum Vent. & $\mathrm{E}$ & Pittosporaceae & 1 & 0,09 \\
\hline Plátano & Platanus acerifolia (Aiton) Willd. & $\mathrm{E}$ & Platanaceae & 1 & 0,09 \\
\hline Cerejeira & Prunus serrulata Lindl. & $\mathrm{E}$ & Rosaceae & 1 & 0,09 \\
\hline Pereira & Pyrus communis L. & $\mathrm{E}$ & Rosaceae & 1 & 0,09 \\
\hline Ráfis & $\begin{array}{c}\text { Raphis excelsa (Thunberg) Henry ex. } \\
\text { Rehder }\end{array}$ & $\mathrm{E}$ & Arecaceae & 1 & 0,09 \\
\hline Carvalho-brasileiro & Roupala brasiliensis Klotz. & $\mathrm{N}$ & Proteaceae & 1 & 0,09 \\
\hline Sena & Senna multijuga (Rich.) Irwin et Barn. & $\mathrm{N}$ & Fabaceae & 1 & 0,09 \\
\hline Aroeira-periquita & Schinus molle L. & $\mathrm{E}$ & Anacardiaceae & 1 & 0,09 \\
\hline Guapuruvu & Schizolobium parahyba (Vell.) Blake & $\mathrm{N}$ & Fabaceae & 1 & 0,09 \\
\hline \multicolumn{4}{|c|}{ TOTAL } & 1059 & 100 \\
\hline
\end{tabular}

Quanto à altura da vegetação, observou-se que 11,2\% dos indivíduos são classificados como de pequeno porte (Figura 2), sendo estes indivíduos constituintes de maciço, tais como dietes, babosa, agave, nandina, rosa e eufórbia. Essas espécies de pequeno porte apresentaram-se em formação de tufos ou mesmo isoladas na área livre da calçada ou ainda, associadas a arbustos ou arbóreas.

A vegetação classificada com porte médio $(38,4 \%)$ ou grande $(50,4 \%)$ é representada por arbustos e arbóreas jovens ou podadas. Tipuana, timbaúva, umbu, álamo, tamareira-das-canárias e canafístulas são as arbóreas que constituem os elementos de maior porte presentes no bairro. Assim, muitas das espécies de grande porte apresentam seus indivíduos jovens, que ainda não apresentam os transtornos típicos dessa vegetação ao meio urbano, tais como danos a calçadas e conflito com a fiação.
As deformações nas espécies arbóreas advêm de vários fatores, e na arborização de ruas e avenidas, a poda é o principal fator de alteração da estrutura da copa. Para o bairro Nossa Senhora das Dores, apenas 25,12\% apresentavam-se classificadas com estrutura normal, ou seja, arbóreas cuja morfologia se apresentou em perfeito estado. Já 74,88\% dos indivíduos observados apresentaram deformações causadas por podas mal conduzidas. As arbóreas cujas estruturas foram drasticamente abaladas pelas podas são $23,62 \%$ do total (Figura 3). Nas árvores de grande porte, como no caso da tipuana, a deformação encontrada na copa deveu-se a aplicação de podas de compatibilização com a rede elétrica. A espécie com maiores deformações foi a extremosa, por causa, principalmente, das intervenções drásticas realizadas pela própria população.

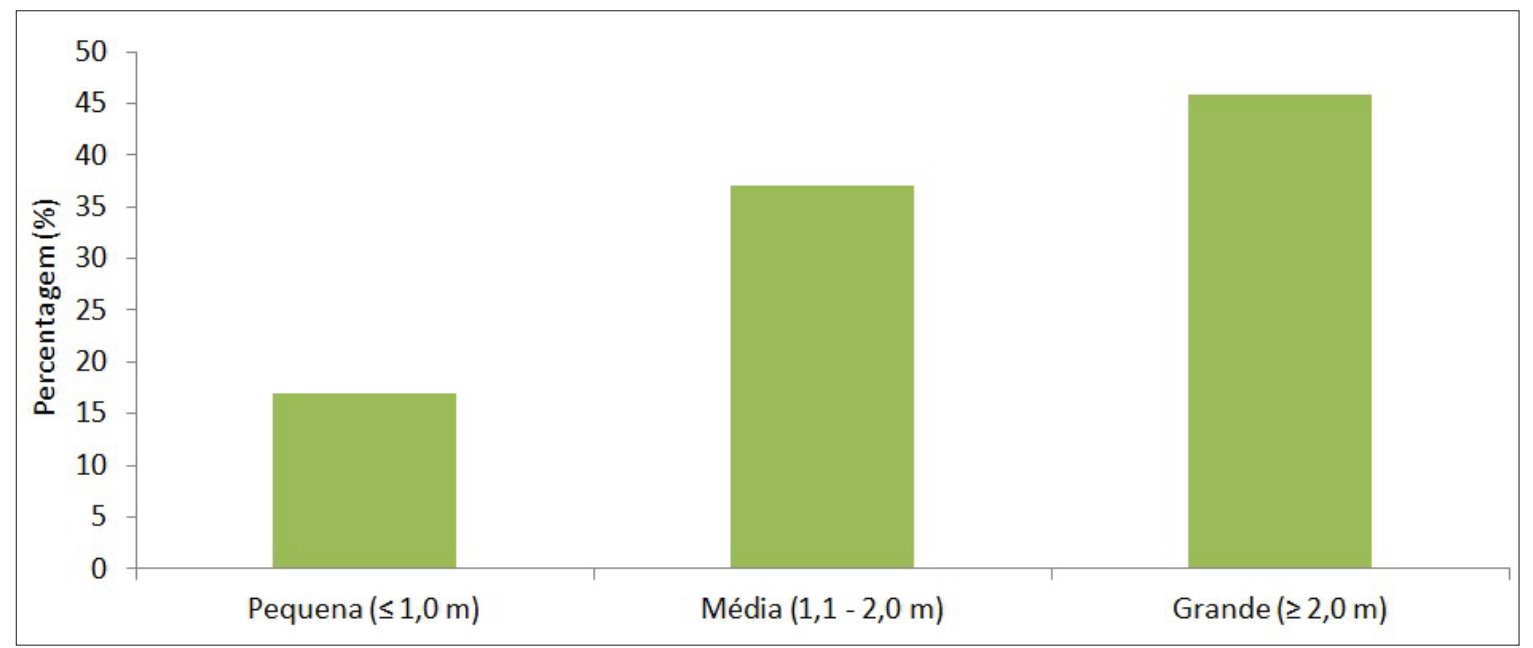

Figura 2. Classes de altura (\%) dos indivíduos ocorrentes na arborização do Bairro Nossa Senhora das Dores. Santa Maria, 2010.

Figure 2. Classes of height (\%) of individuals occurring in afforestation in Nossa Senhora das Dores neighborhood. Santa Maria, RS, 2010. 


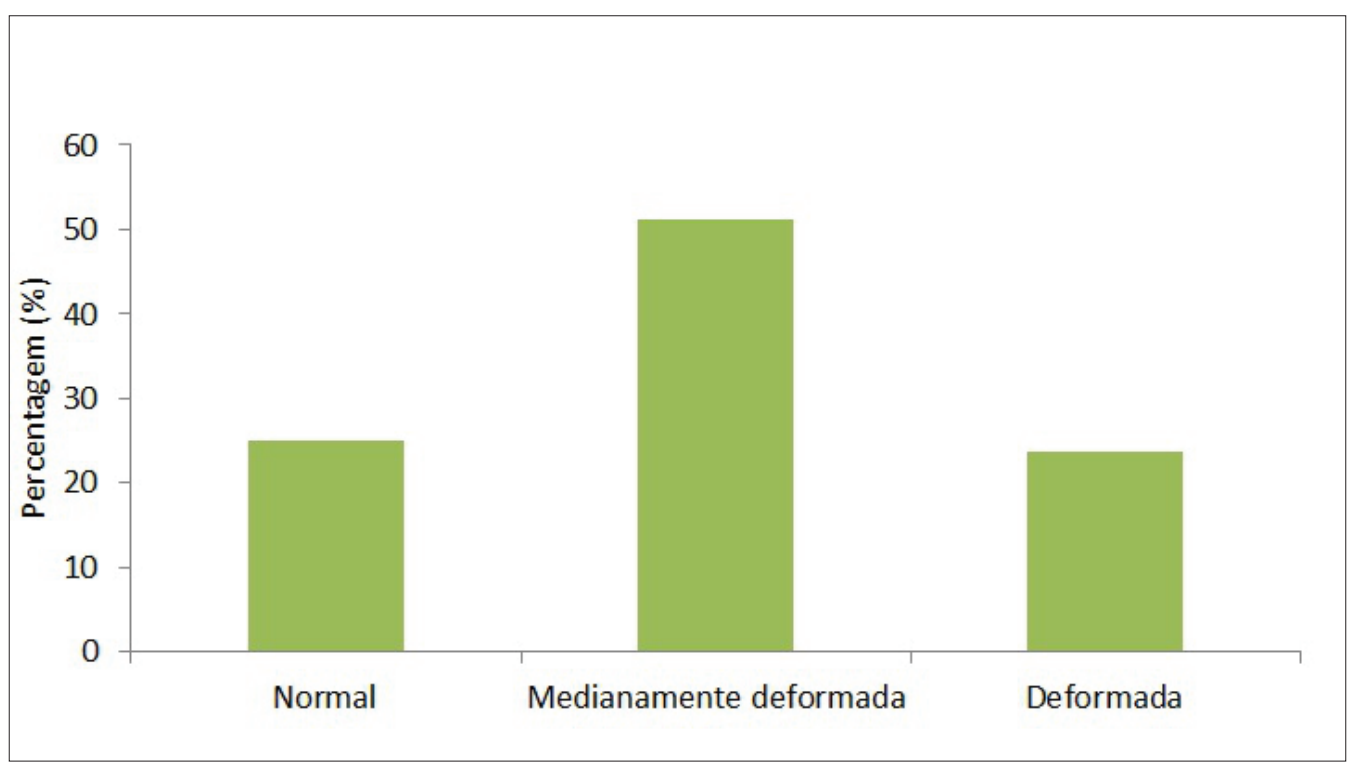

Figura 3. Estado da estrutura da copa de espécies arbóreas ocorrentes na arborização do bairro Nossa Senhora das Dores subdividida em classes (\%). Santa Maria, 2010

Figure 3. Canopy structure state of tree species in afforestation in Nossa Senhora das Dores neighborhood subdivided into classes (\%). Santa Maria, 2010

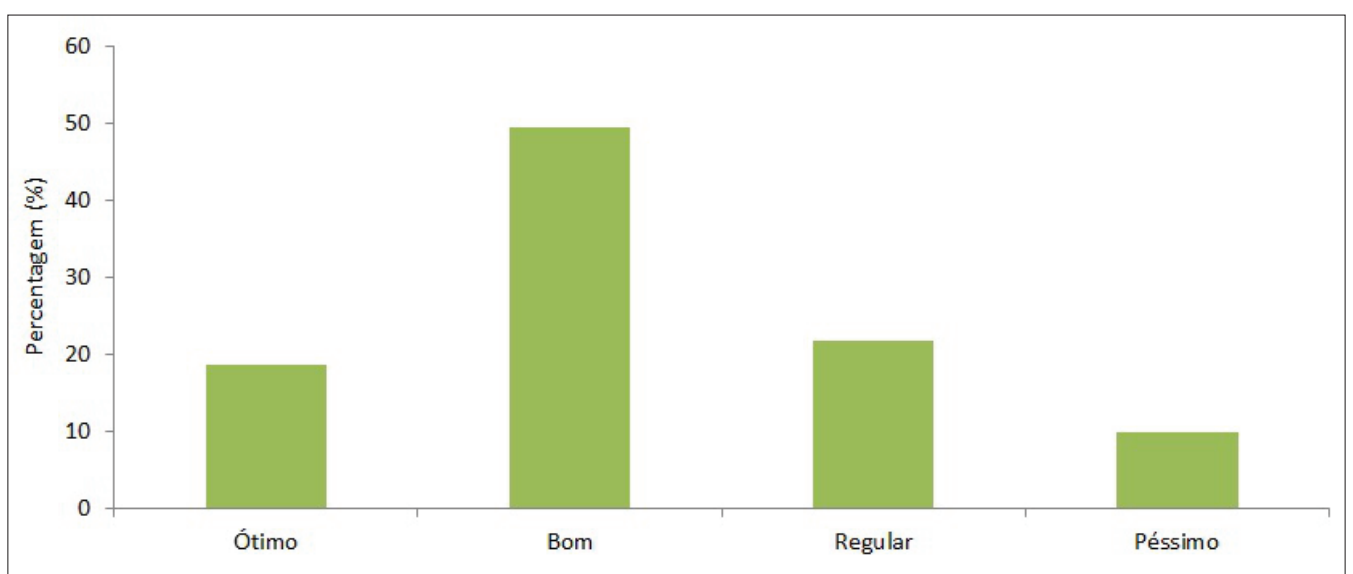

Figura 4. Classes (\%) da situação da vegetação do Bairro Nossa Senhora das Dores, segundo seu aspecto geral. Santa Maria, 2010.

Figure 4. Classes (\%) of the state of vegetation in Nossa Senhora das Dores neighborhood, according to its general appearance. Santa Maria, 2010.

O parâmetro relativo ao aspecto geral das espécies está apresentado na Figura 4, na qual é possível observar que a maioria dos indivíduos apresenta-se em ótimo e bom estado (68,3\%). A vegetação em péssimo estado representa $9,9 \%$ dos indivíduos, e a extremosa constitui a espécie de maior representatividade, seguida do ligustro, especialmente por se tratarem de plantas de idade avançada, já que estas foram as primeiras a serem introduzidas na arborização do bairro.

Em relação aos danos provocados pela vegetação às calçadas, verificou-se que $23,4 \%$ dos exemplares causaram danos aos passeios, devido ao desenvolvimento radicular, sendo que a tipuana foi a espécie que mais provocou esta situação, seguida pelo ligustro, ingá e fícus-benjamina. A extremosa, planta indicada ao paisagismo urbano por não provocar danos às calçadas, apresentou nove exemplares com danos ao passeio, provavelmente porque a área livre para seu desenvolvimento é muito exígua.

Quanto ao conflito da copa com a fiação, verificou-se que $17,5 \%$ dos indivíduos ocorrentes na arborização do bairro apresentam-se nesta situação, em que as árvores de grande porte, como tipuana, uva-do-japão e grevílea, representam as espécies com maior frequência para este problema.

\section{CONCLUSÃO}

Os resultados indicam que as espécies inadequadas existentes nas vias públicas do bairro Nossa Senhora das Dores devem ser substituídas gradativamente por espécies compatíveis com o espaço urbano, a fim de proporcionar a melhoria do ambiente para os habitantes e transeuntes. Sugere-se ainda um plano de arborização e um trabalho de conscientização, que atenda às necessidades do bairro. 


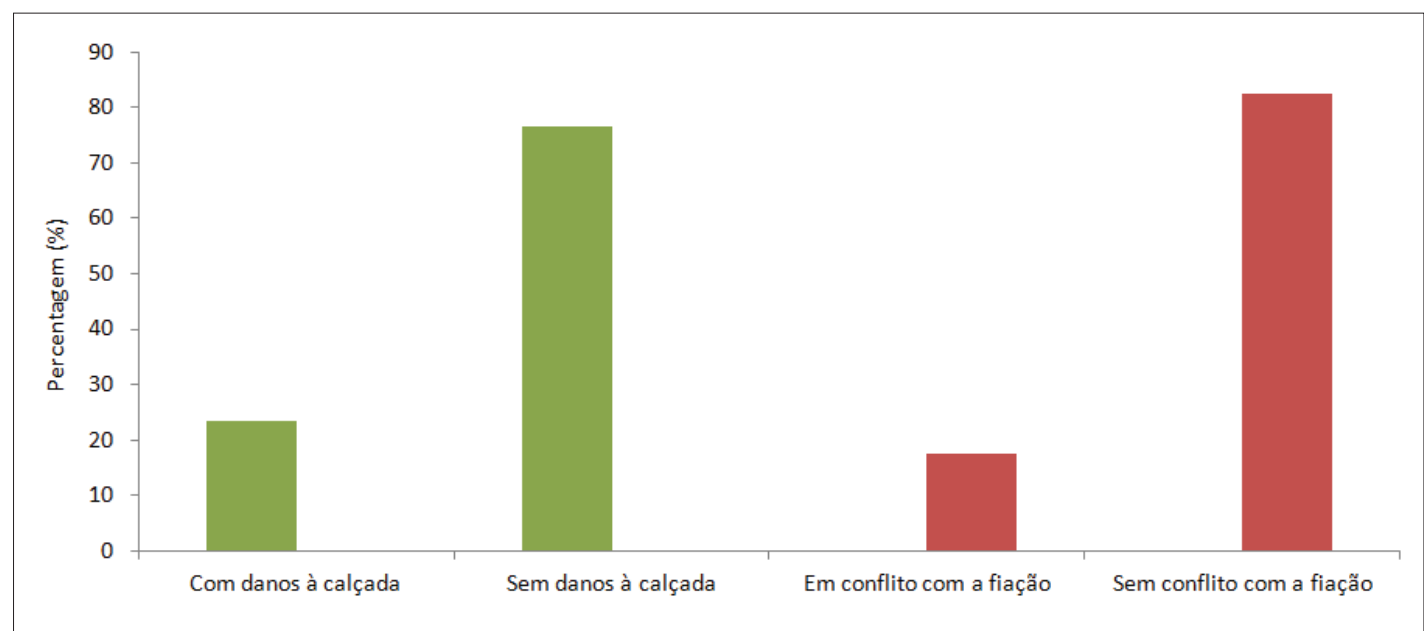

Figura 5. Percentual de interferência da vegetação viária do bairro Nossa Senhora das Dores ao patrimônio público, relativo aos danos às calçadas e em conflito com a fiação. Santa Maria, 2010. Figure 5. Interference percentage of vegetation road in Nossa Senhora das Dores neighborhood public property, damage on the sidewalks and in conflict with the wiring. Santa Maria, 2010.

\section{REFERÊNCIAS}

GONÇALVES, W.; PAIVA, H.N. Árvores para o ambiente urbano. Viçosa: Aprenda Fácil, v.3, 2004. 238p.

MILANO, M.; DALCIN, E. Arborização de vias públicas. Rio de Janeiro: Light, 2000. 226p.

PIVETTA, K.F.L.; SILVA FILHO, D.F. Arborização Urbana. Boletim Acadêmico, série Arborização Urbana. UNESP/ FCAV/FUNEP, Jaboticabal: São Paulo, 2002. 74p.
RIBEIRO, R.C.S.R.; HARDER, I.C.F.; TAVARES, A.R. Avaliação qualitativa e quantitativa da arborização das praças de Vinhedo, SP. Revista Brasileira de Horticultura Ornamental, Campinas, v.12, n.2, p.75-86, 2007.

SILVA FILHO, D.F.; PIZETTA, P.U.C.; ALMEIDA, J.B.S.A.; PIVETTA, K.F.L.; FERRAUDO A.S. Banco de dados relacional para cadastro, avaliação e manejo da arborização em vias públicas. Revista Árvore, Viçosa, v.26, n.5, p.629-642, 2002. 